\title{
CoDAPT: A Concurrent Data And Power Transceiver for Fully Wireless 3D-ICs
}

\author{
Benjamin J. Fletcher*, Shidhartha Das ${ }^{\dagger}$, Terrence Mak* \\ *Department of Electronics and Computer Science University of Southampton, UK. ${ }^{\dagger}$ Arm Ltd, Cambridge, UK. \\ *\{bjf1g13,tmak\}@ecs.soton.ac.uk, ${ }^{\dagger}$ shidhartha.das@arm.com
}

\begin{abstract}
Three dimensional system integration is a promising enabling technology for realising heterogeneous ICs, facilitating stacking of disparate elements such as MEMS, sensors, analogue components, memories and digital processing. Recently, research has looked to contactless 3D integration using inductive coupling links (ICLs) to provide a low-cost alternative to conventional contact-based approaches (e.g. through silicon vias) for 3D integration. In this paper, we present a novel, fully wireless, ICL architecture for Concurrent Data and Power Transfer (CoDAPT) between tiers of a 3D-IC. The proposed CoDAPT architecture uses only a single inductor for simultaneous power transmission and data communication, resulting in high area efficiency, whilst facilitating low-cost, straightforward die stacking. The proposed design is experimentally validated through full wave EM and SPICE simulation and demonstrates capability to communicate data vertically at a rate of $1.3 \mathrm{Gbps} /$ channel (utilising an area of only $0.052 \mathrm{~mm}^{2}$ ) whilst simultaneously achieving power delivery of $0.83 \mathrm{~mW}$, under standard operating conditions. A case study is also presented, demonstrating that CoDAPT achieves an area reduction greater than $1.7 \times$ when compared with existing works, representing an important progression towards ultra low-cost 3DICs through fully wireless stacking.
\end{abstract}

\section{INTRODUCTION}

Three dimensional (3D) system integration is a highly promising 'more-than-Moore' technology that facilitates high density integration by extending planar ICs vertically. A range of different 3D integration methodologies exist, however one of the most prevalent is the use of through silicon vias (TSVs) to interconnect stacked dies. TSVs, however, have been reported to suffer from reliability and yield issues, and their formation requires a number of additional processing stages [1]. This results in TSV-based 3D integration incurring high design and manufacturing costs [2]. To address this, recent research has explored the use of inductive coupling links (ICLs) to transmit data between vertically stacked dies. ICLs allow contactless 3D integration where data is transmitted purely by electromagnetic (EM) coupling between planar inductors fabricated in the back-end-of-line (BEOL) interconnect layers of each die. When adopting ICLs (for 3D integration), existing fabrication processes can be used without alteration, making them an attractive low-cost alternative to TSVs.

One challenge when using ICLs, however, is delivering power between tiers. Whilst existing ICL designs are useful for communicating data between dies, each die must still have its own power supply. This is typically achieved through using wire-bonding (to bond power ground and reset signals to each

Experimental data used in this paper can be found at DOI:10.5258/SOTON/D0728 (https://doi.org/10.5258/SOTON/D0728).

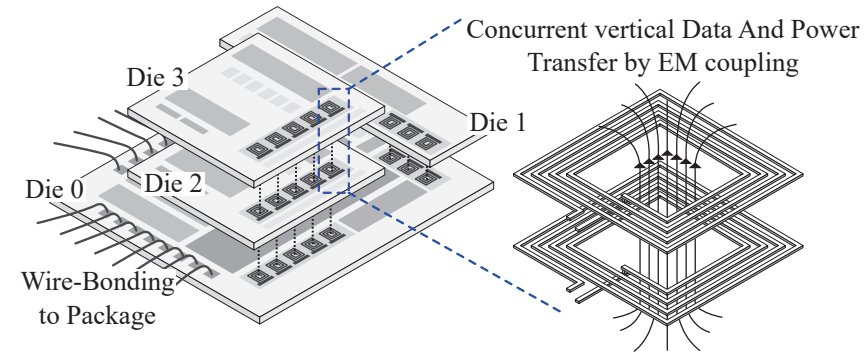

Fig. 1: Exploded illustration of fully wireless 3D-IC enabled by CoDAPT.

stacked tier [3]). For cases where many dies are stacked this becomes complicated, and intricate stacking patterns (such as terraced or spiral-stair stacking [3]) are required. This inflates the cost and complexity of devices, and the addition of wire bonds undermines many of the benefits associated with contactless integration.

In this paper, we present an alternative approach, an ICL architecture that enables Concurrent Data And Power Transmission (CoDAPT) between stacked dies, using a single smallfootprint inductor. The concept of CoDAPT is illustrated in Fig. 1: fully wireless 3D integration, whereby data and power are delivered vertically through a single inductive coupling channel. In achieving this, the novel contributions of this paper can be summarised as follows:

- A bi-phase shift-keying ICL transceiver architecture that achieves vertical data and power delivery concurrently within a 3D-IC.

- In-depth analysis of ICL inductor layouts focussing on the trade-off between power delivery efficiency and bandwidth, resulting in a high-coupling-coefficient $(k=0.36)$, high-bandwidth $(1.3 \mathrm{GHz})$ design.

- Validation of the proposed transceiver and layout demonstrating the capability to communicate data vertically at a rate of $1.3 \mathrm{Gbps} /$ channel whilst simultaneously achieving a power delivery density of $16.0 \mathrm{~mW} / \mathrm{mm}^{2}$. This represents the most area efficient solution available for simultaneous power and data delivery in contactless 3DICs (more than $1.7 \times$ improvement on the state-of-the-art).

- Experimental evaluation of the effects of die-to-die misalignment (between stacked tiers) on the proposed approach, demonstrating that it can tolerate $\pm 28 \mu \mathrm{m}$ of lateral displacement in $x$ and $y$ directions whilst maintaining power delivery performance within $10 \%$ of the optimum. 


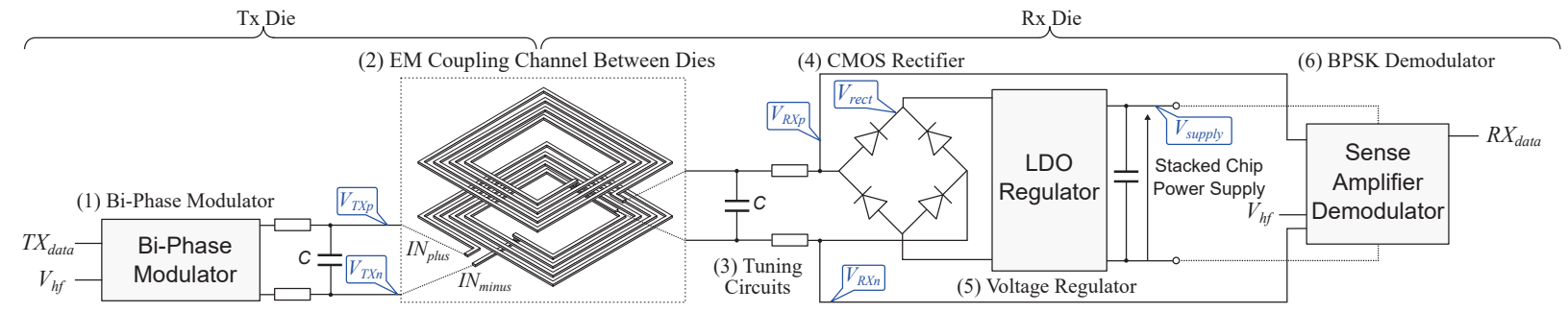

Fig. 2: Schematic diagram illustrating the proposed CoDAPT architecture.

\section{BACKGROUND}

Contactless 3D integration, most notably using near-field inductive coupling to communicate data, has been proposed as a low-cost alternative to TSVs for designing 3D-ICs [2]. In such systems, data is encoded in a series of current pulses which are fed through a planar transmit (TX) inductor, fabricated in the upper BEOL interconnect layers of the transmitting die. These current pulses form a magnetic field, which is intersected by a second inductor fabricated in the receiving (RX) die, as illustrated in Fig. 1. According to the principle of electromagnetic induction, this causes a corresponding current (and hence voltage) to be induced in the recipient inductor which can be de-coded to recover the data.

Three dimensional integrated circuits constructed using this approach are widely reported [4]-[6], however only provide a contactless interface for data delivery between tiers. Because of this, 3D-ICs using ICLs often achieve power delivery through other separate means, the most common being wire-bonding. Wire-bonding is an adequate solution which achieves the goal of circumventing TSVs, however, the stacking arrangements required for such systems are intricate and complex (as outlined above), and undermine some of the benefits of contactless 3D integration which aims to be cheap, simple and robust.

One alternative to wire-bonding, for power delivery, is the use of Highly Doped Silicon Vias (HDSVs), proposed in [2]. HDSVs are vertical channels formed from highly doped wells to deliver power through dies after aggressive thinning. Whilst this is a promising future technology, it is yet to be practically realised and HDSVs will require a substrate thickness less than $5 \mu \mathrm{m}$ [2], likely introducing a number of physical challenges, in addition to significantly increasing the cost, beyond wire-bonded approaches. Another method of transferring power between vertically stacked dies is using wireless power transfer (WPT). Prior works report WPT of between $2.5 \mathrm{~mW}$ [7] and $12.3 \mathrm{~mW}$ [8] per channel within 3DICs. These works, however, use large inductors (separate to those for data transmission) that are often greater than $500 \mu \mathrm{m}$ in diameter [7], meaning that the entire contactless interface (for power and data delivery) can consume up to $1 \mathrm{~mm}^{2}$ of silicon area, or more, which is undesirably large.

\section{Concurrent Power And Data Delivery ARCHITECTURE}

To address this challenge, as discussed in the introduction, the aim of the CoDAPT architecture is to facilitate power and

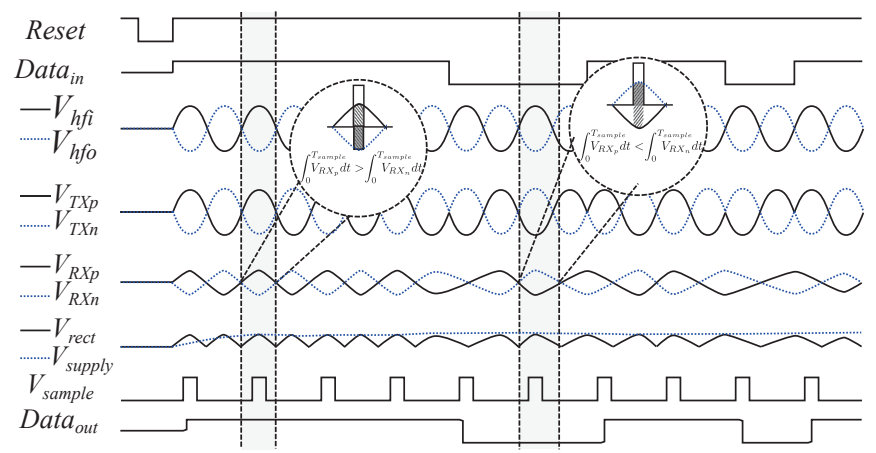

Fig. 3: Operation of CoDAPT: $V_{h f i}$ and $V_{h f o}$ are the in and out-of phase carriers, $V_{\text {rect }}$ is the rectified RX signal, $V_{\text {supply }}$ is the RX supply and $V_{\text {sample }}$ is the SA sample window.

data delivery concurrently using a single inductive channel. To achieve this, continuous bi-phase shift keying (BPSK) modulation will be used to ensure constant power delivery between tiers, irrespective of the TX data stream (unlike prior works which discretise transmission). Fig. 2 shows an overview of the proposed CoDAPT architecture, consisting of: (1) a bi-phase shift keying modulator, (2) the ICL channel, (3) tuning circuits to ensure that the system operates at resonance (to improve efficiency [9]), (4) a CMOS rectifier, (5) an LDO regulator (based upon a band-gap reference) to manage the received power supply for the recipient die, and (6) a senseamplifier (SA) based coherent de-modulator. For the system to work correctly, each of these elements must be carefully designed to maximise power delivery efficiency, whilst still supporting data transmission. The entire system is designed for on-chip integration and will hence facilitate straightforward, low-cost 3D integration where dies can be fabricated, and then stacked, with no additional processing required. The design of each element is documented below.

\section{A. Bi-Phase Transceiver Design}

Fig. 3 illustrates the BPSK modulation scheme proposed for use in this work. The CoDAPT architecture is differential and the modulator operates by selectively driving the in-phase or out-of-phase $\left(180^{\circ}\right.$ shifted) carrier around the coil in accordance with the TX data stream. Fig. 4 illustrates the proposed modulation circuit required to realise this transmission. Here, $V_{h f_{i}}$ is the in-phase high frequency carrier signal, $V_{h f_{o}}$ is the shifted carrier signal, and $I N_{\text {plus }}$ and $I N_{\text {minus }}$ are the driving ports for the ICL channel. The modulation circuits operate at nominal voltage $(1.2 \mathrm{~V})$ to minimise power dissipation, 

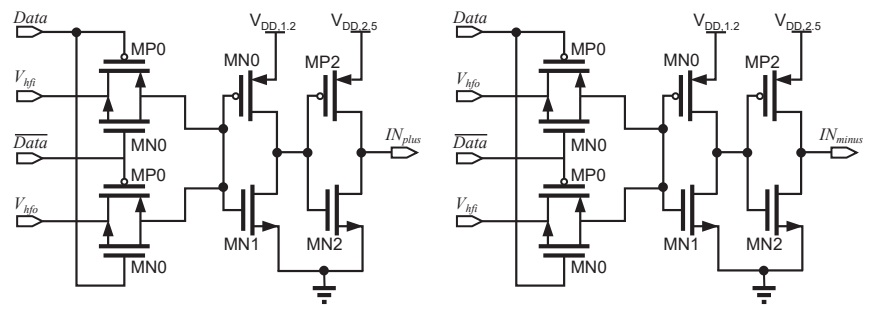

Fig. 4: Schematic diagram of proposed CoDAPT bi-phase keying modulator

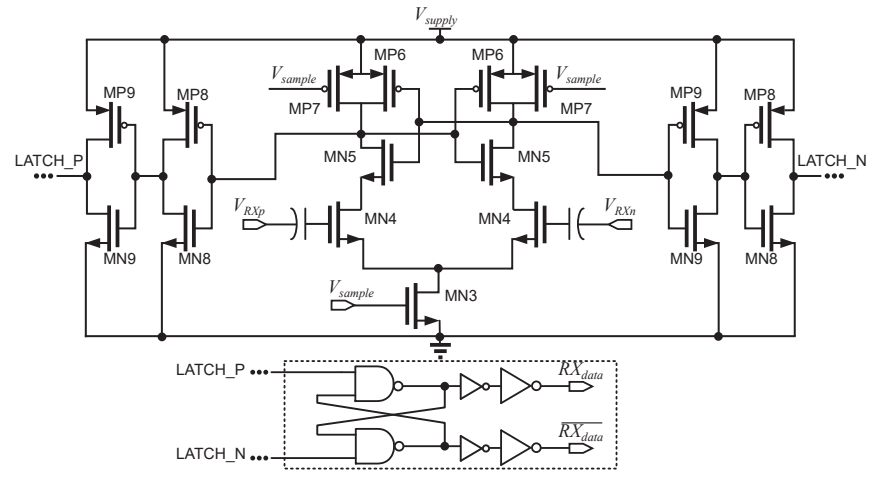

Fig. 5: Schematic diagram of proposed CoDAPT senseamplifier based de-modulator.

however to maximise the RX pulse amplitude the coils are driven by MN2 and MP2 which connect to a $2.5 \mathrm{~V}$ supply. Fig. 5 shows the proposed corresponding demodulator design, based on a sense amplifier (SA) [10]. The operation of this circuit is illustrated in the dashed circles in Fig. 3. The SA will sample the differential received signal, $V_{R X_{n}}, V_{R X_{p}}$ in the interval $T_{\text {sample. }}$. In the case the at a ' 1 ' has been transmitted

$$
\int_{0}^{T_{\text {sample }}} V_{R X_{p}} d t>\int_{0}^{T_{\text {sample }}} V_{R X_{n}} d t
$$

and hence, LATCH_P will be pulled high by the differential pair (MN4) and a ' 1 ' will be latched at the output. Conversely, as shown on Fig. 3, if a ' 0 ' has been transmitted

$$
\int_{0}^{T_{\text {sample }}} V_{R X_{p}} d t<\int_{0}^{T_{\text {sample }}} V_{R X_{n}} d t
$$

as the sampling period aligns with a peak in the $V_{T X_{n}}$ signal. In this case, the differential pair will pull LATCH_N high, and hence a ' 0 ' will be received. The sensitivity of this sense amplifier is determined by the width of MN4.

One of the main sources of power dissipation in CMOS circuits is dynamic power consumption. Because of this, a trade-off between power delivery and power efficiency exists within the transceiver: When operating at a higher frequency, the variation in magnetic flux will be greater, allowing more power delivery between coils. When operating at higher frequency, however, the dynamic switching power dissipation will be much greater. To strike a balance between these two factors, a carrier frequency of $2 \mathrm{GHz}$ was selected. This represents a sufficiently high frequency for meaningful power transfer,

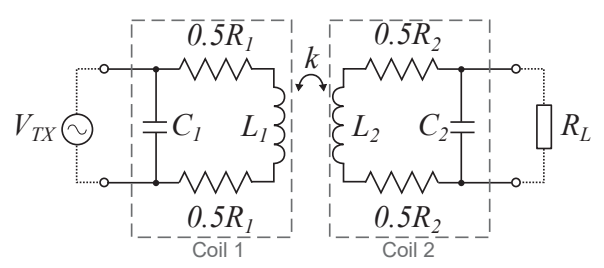

Fig. 6: Equivalent circuit model of an ICL channel [13] which assumes that each coil can be accurately modelled by its resistance $\left(R_{i}\right)$, capacitance $\left(C_{i}\right)$, inductance $\left(L_{i}\right)$.

(a)

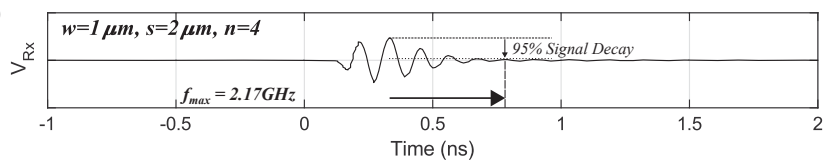

(b)

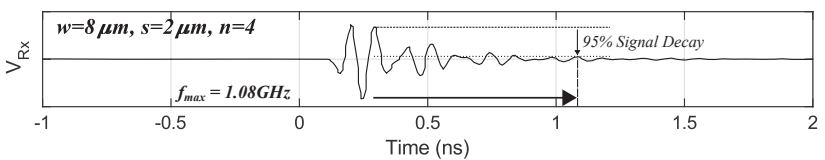

(c)

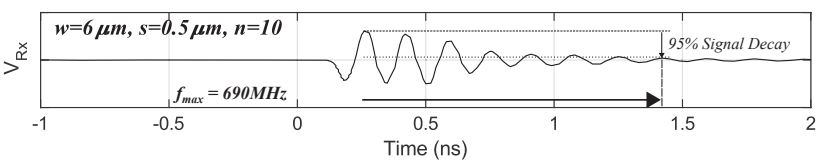

Fig. 7: Illustration of the effects of physical layout parameters (track width, $w$, track spacing, $s$, and number of turns, $n$ ) on the RX voltage signal for 3 example cases.

whilst remaining in the operating tolerances of the $65 \mathrm{~nm}$ technology (used for implementation in this work), hence minimising dynamic power dissipation.

\section{B. Inductive Channel Design}

The second element in the CoDAPT architecture is the inductive coupling channel itself, consisting of two coupled planar inductors. Power transmission between two stacked inductors is optimised when their layouts are congruent [11]. Therefore, two congruent square spiral inductors are considered in this paper (as square inductors offer the highest inductance per unit area [12]). As the aim of CoDAPT is to enable concurrent wireless data and power delivery with a low area overhead, a maximum inductor area of $0.04 \mathrm{~mm}^{2}$ was selected, representing a $2.5 \times$ reduction compared to stateof-the-art works exploring WPT in 3D-ICs [8].

Fig. 6 shows an equivalent circuit model of an inductive coupling channel where each inductor, $i$, is modelled by its self-capacitance $C_{i}$, resistance, $R_{i}$, inductance, $L_{i}$ and the EM coupling coefficient $k$ that exists between the inductor pair. From this model, an equation for the power delivery efficiency, $\eta_{\text {pow }}$, (power-out/power-in) of a given link can be derived in terms of $C_{i}, R_{i}, L_{i}, k$ and the link's excitation frequency. This is shown in Footnote 1. As the channel in this work will be used to transmit data (in addition to power), consideration must also be given to the coil's RC parasitics. Whilst coil layouts with high numbers of turns, and small intra-turn spacing may maximise $\eta_{\text {pow }}$, these result in high self-capacitance which can cause inter-symbol interference

$$
{ }^{1} \eta_{\text {pow }}=\omega^{2} M^{2} R_{L} /\left\{R_{L}{ }^{2}\left[\left(\omega^{4} C_{2}{ }^{2}\left(R_{2} M^{2}+R_{1} L_{2}{ }^{2}\right)\right)+\omega^{2}\left(R_{1}\left(C_{2}^{2} R_{2}^{2}-2 C_{2} L_{2}\right)+\left(L_{2}^{2} R_{1}+M^{2}\left(R_{L}+R_{2}\right)\right)\right)\right]+R_{1}\left(R_{L}+R_{2}\right)^{2}\right\}(3)
$$




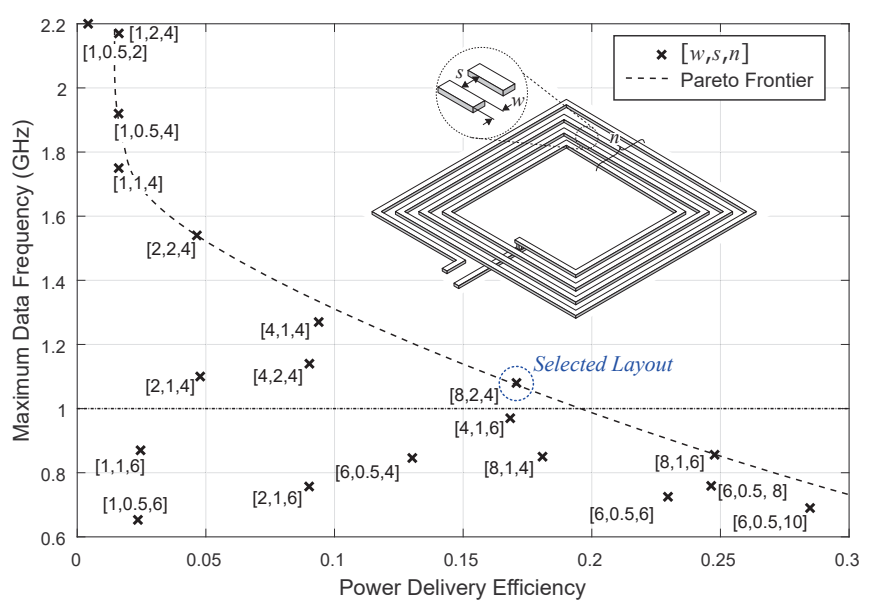

Fig. 8: Trade-off between power efficiency and maximum operating frequency, including pareto-optimal frontier (only a small sample of layouts are presented here for clarity).

(ISI) when transmitting data.

This effect is illustrated in Fig. 7. Here, three transient simulations are presented illustrating the received voltage signal (responding to a square transmit pulse at 0ns) when transmitted through an ICL channel where the inductor layout parameters: track width $(w)$, track spacing $(s)$, and number of turns $(n)$, vary between (a) and (c). As can be observed, the layout in Fig. 7 (a) has fewer turns and a large turn spacing meaning parasitics are low, and the pulse smearing is minimal. Conversely, Fig. 7 (c) uses 10 turns with a small turn-spacing, meaning that the self-capacitance of the coil, $C_{i}$, is high. As a result the signal duration is much longer.

Fig. 8 illustrates the trade-off between power delivery efficiency, $\eta_{\text {pow }}$, and the maximum data bandwidth supported by the link for a small number of selected layouts. The maximum data bandwidth was calculated from the inverse of the $95 \%$ decay point shown in Fig. 7. To select an inductor layout for use in CoDAPT, the pareto frontier was added to Fig. 8 and an arbitrary minimum bandwidth of $1 \mathrm{GHz}$ was defined, to remain competitive with prior ICL data links [6]. The layout with the highest power delivery efficiency, whilst meeting this target, was then selected with $w=8 \mu \mathrm{m}, s=2 \mu \mathrm{m}$ and $n=4$. This corresponds to a resistance of $5.48 \Omega, k$, of 0.36 and a capacitance of $36.7 \mathrm{fF}$ when mapped to the model in Fig. 6.

\section{Tuning Circuit Design}

Although the generation of this optimised layout includes consideration for the link's operating frequency, the resistance $R$ and capacitance $C$ of the coil may only be selected from a finite number of $R C$ combinations that correspond to real, physical inductors. Because of this, the performance of the system can be 'fine-tuned' by adding a series-parallel tuning circuit before and after the coil, as shown in Fig. 2. In order to ensure maximal power delivery between tiers within the 3D-IC, the link should operate at resonance [9]. In this case, resonant operation also ensures that the received voltages are sufficient to allow direct LDO regulation (to the nominal $1.2 \mathrm{~V}$ ) without requiring an additional boost converter. In

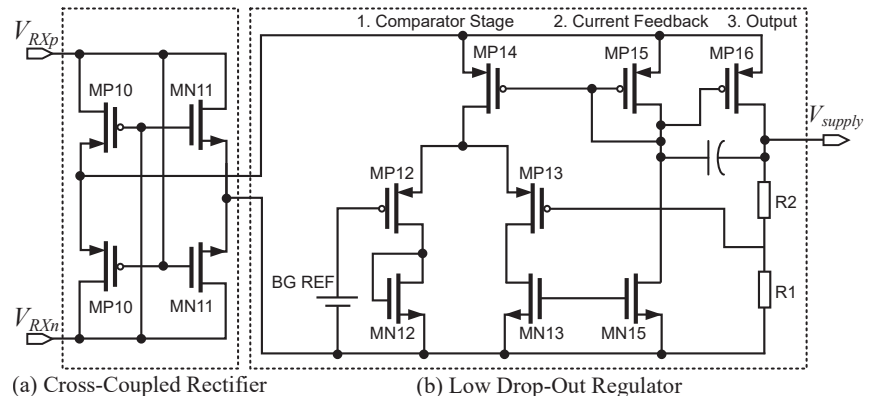

(a) Cross-Coupled Rectifie

(b) Low Drop-Out Regulato

Fig. 9: Schematic diagram of (a) Cross-coupled CMOS rectifier and (b) Low drop-out voltage regulator circuit.

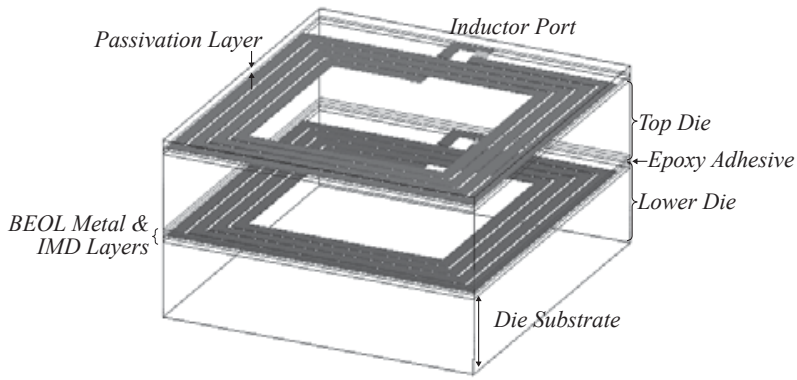

Fig. 10: 3D Illustration of stackup used for EM simulation.

this implementation, no alteration is made to the resistance, however a small tuning capacitor $C$ is added in parallel with the coil, as shown on Fig. 2, to achieve resonance at $2 \mathrm{GHz}$.

\section{Rectifier and Low Drop-Out Regulator Design}

To rectify the received BPSK signal (and hence recover the transmitted power), a CMOS cross-coupled rectifier is used as illustrated in Fig. 9 (a). Work by Han et al. [9] presents extensive comparison of available on-chip rectifier solutions for this style of application, concluding that a cross-coupled rectifier can provide the highest efficiency [9]. Following this rectification stage, a low-drop-out regulator is incorporated in order to regulate the power supply in the recipient die. This is shown in Fig. 9 (b). The regulator operates on the principal that as the voltage $\left(V_{\text {supply }}\right)$ rises, the comparator stage (2) (that compares $V_{\text {supply }}$ to the band-gap reference, BG REF) will cause the gate voltage of MP16 to increase, hence increasing the resistance across it. This will have the effect of limiting the supply voltage to the pre-defined value (set by $R_{1}, R_{2}$ ). The size of the storage buffer (placed at the output, $V_{\text {supply }}$ ) for experiments in this paper was selected to be $1 \mathrm{pF}$.

\section{Results And Evaluation}

\section{A. Experimental Set-Up}

Combining each of these components, the proposed architecture was experimentally validated using commercial simulation tools. The layout was imported to Ansys HFSS for EM simulation of the channel, using the stack-up in Fig. 10. Here, the default metal (and corresponding dielectric) layer thicknesses associated with the TSMC $65 \mathrm{~nm}$ technology are used (passivation $=2 \mu \mathrm{m}$, metal $=0.9 \mu \mathrm{m}$ ) and the epoxy thickness is assumed to be $2 \mu \mathrm{m}$. The only additional processing assumed is wafer thinning to $50 \mu \mathrm{m}$, in-line with realistic 


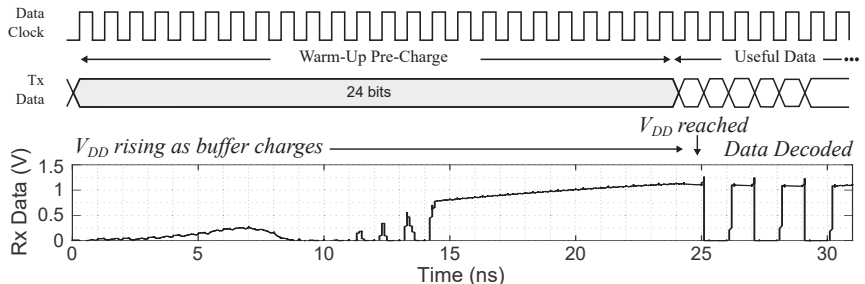

Fig. 11: Transient simulation of CoDAPT start-up sequence.

\begin{tabular}{c|c}
\hline \hline METRIC OF PERFORMANCE & VALUE \\
\hline Area (Including Inductors) & $0.052 \mathrm{~mm}^{2}$ \\
Bandwidth & $1.3 \mathrm{GHz}$ \\
Bit Error Rate (at 1Gbs) & $<10^{-9}$ \\
Latency (at 1Gbs) & $1 \mathrm{~ns}$ \\
Warm-Up Period (at 1Gbs) & $24 \mathrm{bits}$ \\
Peak Power Delivery (per channel) & $2.1 \mathrm{~mW}$ \\
Average Power Delivery (per channel) & $0.83 \mathrm{~mW}$ \\
Power Delivery Density & $16 \mathrm{~mW} / \mathrm{mm}^{2}$ \\
\hline \hline
\end{tabular}

TABLE I: Tabular summary of CoDAPT's performance.

fabrication capabilities. A fitted broadband SPICE model of the channel was exported for simulation of the link in $\mathrm{H}$ SPICE (with the above outlined circuits). Results from these simulations are presented below.

\section{B. Results}

1) Start-Up: Initially, the start-up behaviour of the CoDAPT system was assessed. As the recipient die is powered directly from the communicated data signal when using CoDAPT, there is a small period of time where the energy buffer must charge. Fig. 11 shows the transient performance of the proposed system during this time. Here, a current sink of $0.5 \mathrm{~mA}$ (representing extraneous circuits in the recipient die) is applied. The warm-up period required to ensure robust operation of the ICL data transceiver under these conditions was determined to be approximately $24 \mathrm{~ns}$ ( 24 bits at $1 \mathrm{Gbps}$ ).

2) Data Delivery Performance: After the warm-up period has elapsed, this transceiver was found to exhibit a maximum bandwidth of $1.3 \mathrm{Gbps}$, slightly larger than the theoretical maximum suggested on Fig. 8. The bit error rate (BER) of the proposed transceiver when operating at the target frequency $(1 \mathrm{GHz})$ was found to be $<10^{-9}$. The latency, at the same testing frequency, was determined as 1 clock cycle.

3) Power Delivery Performance: The stability of the supply voltage when using the CoDAPT architecture was then assessed. For normal operation (assuming the transmission of an equiprobable random binary bit sequence) the maximum deviance in the recovered supply voltage $(1.2 \mathrm{~V})$ was measured to be $7 \%$. The instantaneously delivered power reached $2.1 \mathrm{~mW}$ under ideal conditions, however the average power for typical operation was determined to be $0.83 \mathrm{~mW}$ (as there is a slight dependency between the delivered power and the TX datastream $)^{2}$. These metrics are summarised in Table I.

The power and area overheads of the CoDAPT approach were also evaluated. To ascertain the silicon footprint of the approach, physical layout of the CoDAPT transceiver was

${ }^{2}$ Quoted figures are inclusive of the CoDAPT circuitry power dissipation (including data recovery) and carrier signal generation.

\begin{tabular}{c|c|c}
\hline \hline COMPONENT & POWER & $\begin{array}{c}\text { AREA } \\
\text { (65nm technology) }\end{array}$ \\
\hline TX/RX Inductor & N/A & $0.04 \mathrm{~mm}^{2}$ \\
Transmitter & $6.59 \mathrm{~mW}$ & $840 \mu \mathrm{m}^{2}$ \\
Receiver, Demodulator & $2.47 \mu \mathrm{W}$ & $400 \mu \mathrm{m}^{2}$ \\
Receiver, Regulation & $0.661 \mathrm{~mW}$ & $11600 \mu \mathrm{m}^{2}$ \\
Total & $7.16 \mathrm{~mW}$ & $0.052 \mathrm{~mm}^{2}$ \\
\hline \hline
\end{tabular}

TABLE II: Itemised summary of CoDAPT Overheads.
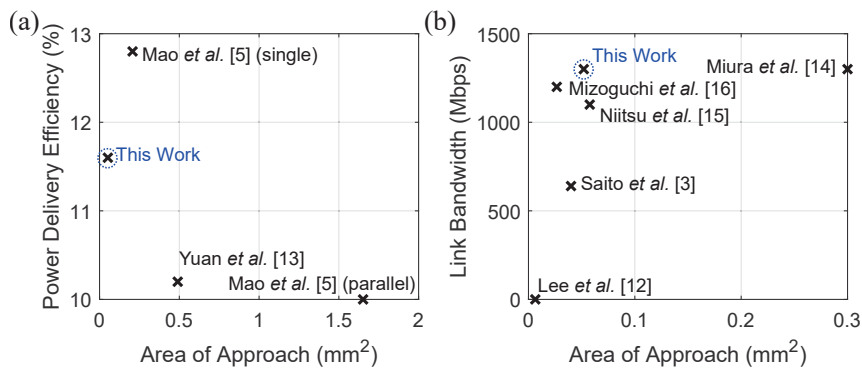

Fig. 12: Comparison of CoDAPT's performance against (a) WPT-specific prior works, and (b) data-specific ICL works.

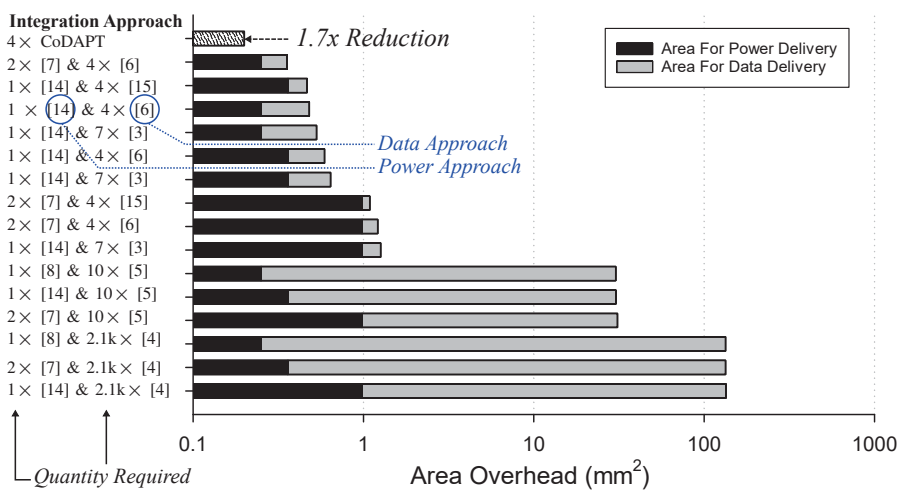

Fig. 13: Area overhead of existing data and power approaches compared with CoDAPT for the assumed integration scenario.

performed using TSMC 65nm LP CMOS technology. The itemised results are shown in Table II.

\section{Advantages of the CoDAPT Approach}

As discussed in the introduction, CoDAPT enables ultralow cost 3D integration, where no additional processing is required due to the fact that both power and data are delivered wirelessly. Previously, fully wireless 3D integration could only be realised using combinations of WPT schemes and data ICLs, resulting in significant area overhead. CoDAPT addresses this by combining power and data delivery in the same circuits.

1) Comparison With Existing Work: Fig. 12 (a) compares the power delivery efficiency of the proposed approach against other works exploring WPT between stacked dies. Despite the fact that CoDAPT supports data transmission in addition to WPT (which is untrue of other approaches in the figure), CoDAPT is very competitive providing high efficiency (11.6\%) with low silicon overhead. Fig. 12 (b) plots bandwidth against area to compare CoDAPT to existing data ICL implementations. Again, CoDAPT is highly competitive achieving the highest bandwidth-per-unit-area using the BPSK scheme. 


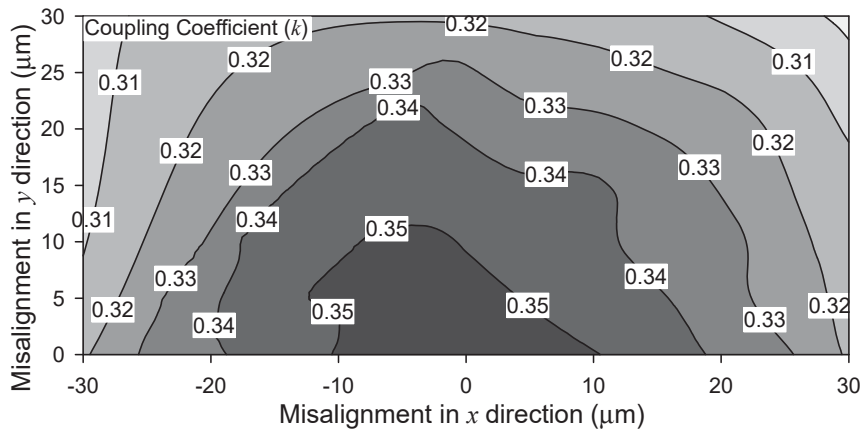

Fig. 14: Effects of lateral die-to-die misalignment on channel coupling coefficient $(k)$.

As concurrent power and data delivery in 3D-ICs is a new concept, CoDAPT can only be compared fairly with combinations of existing data ICLs, and WPT schemes to achieve the same overall net effect. Therefore, for more indepth comparison, Fig. 13 compares the area overhead of CoDAPT with combinations of data-ICL and WPT approaches assuming a use-case scenario of two stacked CPU systems communicating vertically through a 64-bit, $50 \mathrm{MHz}$ data-bus. Modelling each CPU as an Arm M0+ MCU results in a power delivery requirement of approximately $3.0 \mathrm{~mW}$ [16] alongside the data bandwidth requirement of $3.2 \mathrm{Gbps}$. To meet this specification using CoDAPT requires 4 links, resulting in an area overhead of $0.208 \mathrm{~mm}^{2}$. Fig. 13 shows the area overheads of contactless power and data delivery approaches that can be also combined together to meet this specification. From the figure, it can be observed that CoDAPT outperforms each approach in terms of area efficiency, by at least $1.7 \times$ through concurrent data and power transmission, demonstrating that CoDAPT is successful in achieving its aim.

\section{Tolerance to Lateral Misalignment}

Finally, this sub-section evaluates the performance CoDAPT when misalignment exists between stacked tiers. Fig. 14 illustrates the influence of lateral die-to-die misalignment on the coupling coefficient, $k$ (c.f. Fig. 6). Fig. 15 translates these $k$ values into power delivery performance values, expressed as a percentage of the average perfectly-aligned case $(0.83 \mathrm{~mW})$. Setting a target performance tolerance of $\pm 10 \%$, results show that the proposed design allows $\pm 28 \mu \mathrm{m}$ of lateral misalignment in $x$ and $y$ directions (equating to a total offset of $39.6 \mu \mathrm{m}$, almost half of the coil's radius) whilst remaining within this target. Additionally, the proposed architecture can tolerate up to $37 \mu \mathrm{m}$ of lateral misalignment in both directions, before significant degradation in data-transmission performance. When compared to the use of TSVs, this represents an order-of-magnitude improvement, as TSVs typically demand sub-micron alignment accuracy [17].

\section{Conclusions}

This paper presents a novel fully wireless ICL transceiver (CoDAPT), for 3D integration, where data and power can be concurrently delivered through a single channel. Thorough evaluation demonstrated that CoDAPT achieves a data-rate

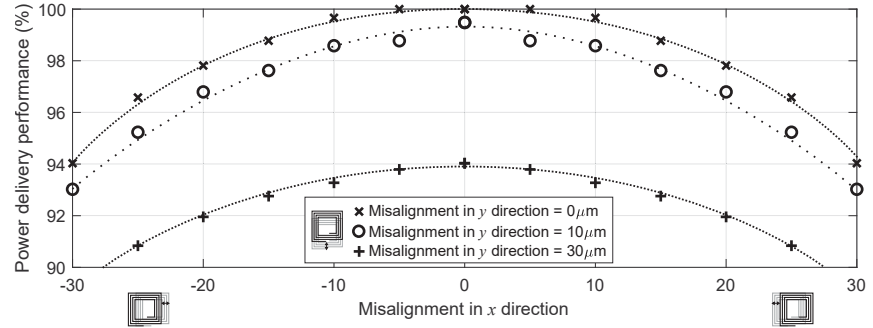

Fig. 15: Effects of lateral die-to-die misalignment on power delivery (normalised to $0.83 \mathrm{~mW}$ ).

of $1.3 \mathrm{Gbps}\left(\mathrm{BER}<10^{-9}\right)$ whilst simultaneously transferring $0.83 \mathrm{~mW}$ of power per channel under typical operating conditions. For the integration scenario discussed in this paper, the area savings when using CoDAPT were in excess of $1.7 \times$ compared to the state-of-the-art. Results also demonstrate tolerance of $\pm 28 \mu \mathrm{m}$ lateral die-to-die stacking misalignment, representing an important progression towards low-cost fully wireless $3 \mathrm{D}$ integration.

\section{REFERENCES}

[1] J. H. Lau, "TSV manufacturing yield and hidden costs for 3D IC integration," in Proc. 60th Electronic Components and Technology Conference (ECTC), June 2010, pp. 1031-1042.

[2] D.Ditzel and T. Kuroda, "Low-cost 3D chip stacking with ThruChip wireless connections," in IEEE Hot Chips 26 Symp. (HCS), Aug 2014.

[3] M. Saito et al., "A $2 \mathrm{~Gb} / \mathrm{s} 1.8 \mathrm{pJ} / \mathrm{b} / \mathrm{chip}$ inductive-coupling through-chip bus for 128-die NAND-Flash memory stacking," in IEEE Int. Solid-State Circuits Conf. (ISSCC), Feb 2010, pp. 440-41.

[4] C. Lee et al., "Transceiver with inductive coupling for wireless chip-tochip communication using a 50-nm digital CMOS process," Microelectronics Journal, vol. 44, no. 9, pp. 852-9, 2013.

[5] N. Miura et al., "A high-speed inductive-coupling link with burst transmission," IEEE J. of Solid-State Cir., vol. 44, no. 3, pp. 947-55, Mar 2009

[6] K. Niitsu et al., "An inductive-coupling link for 3D integration of a 90nm CMOS processor and a 65nm CMOS SRAM," in IEEE Int. Solid-State Circuits Conf. - Digest of Technical Papers, Feb 2009, pp. 480-481.

[7] K. Onizuka et al., "Chip-to-chip inductive wireless power transmission system for sip applications," in IEEE Custom Integrated Circuits Conf., Sept 2006, pp. 575-578.

[8] W. Mao et al., "Analysis and design of high performance wireless power delivery using on-chip octagonal inductor in 65-nm CMOS," in IEEE Int. System-on-Chip Conf. (SOCC), Sept 2015, pp. 401-405.

[9] S. W. Han, "Wireless interconnect using inductive coupling in 3D-ICs," Ph.D. dissertation, The University of Michigan, 2012.

[10] B. Nikolic et al., "Sense amplifier-based flip-flop," in IEEE Int. SolidState Circuits Conf (ISSCC), Feb 1999, pp. 282-283.

[11] B. J. Fletcher, S. Das, and T. Mak, "A high-speed design methodology for inductive coupling links in 3D-ICs," in Design Automation and Test in Europe (DATE), 2018.

[12] S. Mohan et al., "Simple accurate expressions for planar spiral inductances," IEEE J. Solid-State Circuits, vol. 34(10), pp. 1419-24, 1999.

[13] N. Miura et al., "Analysis and design of inductive coupling and transceiver circuit for inductive inter-chip wireless superconnect," IEEE J. of Solid-State Circuits, vol. 40, no. 4, pp. 829-837, Apr 2005.

[14] Y. Yuan et al., "Chip-to-chip power delivery by inductive coupling with ripple canceling scheme," Jap. J. of Applied Phys., vol. 47, p. 2797, 2008.

[15] D. Mizoguchi et al., "A 1.2Gb/s/pin wireless superconnect based on inductive inter-chip signaling (iis)," in IEEE Int. Solid-State Circuits Conf., Feb 2004, pp. 142-517 Vol.1.

[16] "Cortex-M0+," https://developer.arm.com/products/processors/cortexm/cortex-m0-plus, accessed: 2018-04-15.

[17] K. Tu, "Reliability challenges in 3D IC packaging technology," Microelectronics Reliability, vol. 51, no. 3, pp. 517 - 523, 2011. 